Original Article

\title{
Assessment of Frailty Preoperatively and Loss of Independence in Elderly Surgical Patients
}

\author{
Shams-ud-Din ${ }^{1}$, Ishfaq Ahmed ${ }^{2}$ \\ ${ }^{1}$ Islamabad International Hospital and Research Center (IIHRC), ²Quaid e Azam International Hospital, \\ Islamabad - Pakistan
}

\section{ABSTRACT}

Objective: This study was aimed to determine preoperative frailty assessment screening on postoperative morbidity and postoperative loss of independence (LOI).

Materials \& Methods: A total of 100 patient's age 65 years and older were included in this study. Edmodium Frail Scale (EFS) was used for the assessment of frailty. The surgical procedure was categorized on operative severity score, and Hierarchical Condition Category (HCC). The Chi-square test was applied to see the significant/insignificant differences between different stratified groups of patients.

Results: The mean age of the patients was 68 years. There were $63 \%$ male and $37 \%$ female patients. Maximum patients $(78 \%)$ who had no LOI were in age less than 80 years. $11 \%$ of patients who had LOI were only $11 \%$. There existed a significant difference ( $p$-value: 0.004 ) between age groups (greater than/less than 80 years) of patients and presence/absence of loss of independence (LOI). After EFS assessment, 32\% of patients had limited mobility, $22 \%$ had weight loss, $19 \%$ reported depression, $9 \%$ of patients were socially dependent and $18 \%$ reported cognitive impairment. In $40 \%$ of patients, the OSS was 3 or higher, and $60 \%$ had OSS between $1-2$. Post surgically, $15 \%$ of patients experienced a loss of dependence. The OSS was higher in patients with LOI as compared to patients without LOI.

Conclusion: The results suggest that frail patients older than 78 years and OSS $>3$ had a more increased risk of postsurgical LOI. The patients with EFS $>5$ and OSS $>3$ had more LOI. EFS analysis explained that those patients with restricted mobility, depression, and dependent socially had increased LOI. Higher patient dependency before surgery corresponds to poor outcomes compared to patients who do not have a loss of their independence before surgery.

Keywords: loss of independence (LOI), Edmonton Frail Scale (EFS), Hierarchical Condition Category (HCC), Operative Severity Score (OSS).

Corresponding Author: Shams-ud-Din

Islamabad International Hospital and Research Center

(IIHRC), Islamabad - Pakistan

Email: asz-9-0@hotmail.com
Date of Submission: 20-08-2021

Date of Revision: 09-09-2021

Date of Acceptance: 20-09-2021

Date of Online Publishing: 30-09-2021

Date of Print: 30-09-2021

DOI: $10.36552 /$ pjns.v25i3.576 


\section{INTRODUCTION}

The detailed discussion on the preoperative clinical status of older patients (65 years and above) guided the post-operative outcomes regarding morbidity and mortality. ${ }^{1-3}$ Approximately more than 50 percent of operations globally are performed on patients of age more than 65 years. ${ }^{1,3,4}$ Frailty in the population older than 65 years is between 10 $37 \% .3,9,12$ Older patients are more prone to develop anesthesia and operative complications in the postoperative period. ${ }^{5,7,9}$ Increased surgical complications and lengthy hospital stays are reported in older frail patients. ${ }^{1,3,5}$ Those frail patients who develop loss of independence (LOI) need trained rehabilitation centers in the postsurgical period when they discharge from primary hospital. ${ }^{3,5,6}$ Since the emergence of the global pandemic of COVID 19, these rehabilitation centers are very prone to this viral infection with catastrophic outcomes. ${ }^{1,2,11}$ Currently, there was a need for frailty assessment guidelines in the aging population of the World, It was incorporated by the American College of Surgeons together with the American Geriatrics Society in the year 2013. A 67-point instrument has been explained for this vulnerable old age frail patients. ${ }^{3,7-9}$ The instrument points were divided into two types, the physical frailty phenotype and deficit accumulation model. ${ }^{7}$ The physical frailty phenotype is composed of physical ambulation, walking speed and grip strength, endurance, and lean body mass. ${ }^{5}$ The deficit accumulation is characterized by multimorbidity. ${ }^{9,11}$ The assessment of cognition and psychosocial statuses were scantly emphasized in most of the instruments., ${ }^{8,12}$ The Edmodium Frail Scale (EFS) was then started to utilize for the assessment of frailty preoperatively in 2018. The EFS includes an 11 - point scale, 9 of which are self-reporting points. This can assess psychosocial and cognition in the evaluation of frailty.
Frailty is most common in the elderly, and this population is rising at an alarming rate across the world. In 2015, an estimated 8.5 percent of the global population (also known as the elderly) was 65 years old or older. By 2030, this is anticipated to have grown to more than $12 \%$, and by 2050 , it is expected to have risen to more than $17 \%$. Conversely, the extent of people 20 years and more youthful is projected to stay static throughout a similar period. In the USA, more seasoned grown-ups address $15 \%$ of the absolute populace; this rate is projected to increment to $21.4 \%$ by 2050 . In 2012, notwithstanding just representing $12 \%$ of the populace, more established patients represented $35 \%$ of all medical clinic confirmations. This accomplice additionally had an altogether longer normal medical clinic stay and an expanded normal expense for each stay. Notwithstanding the absence of the highest quality level functional definition, slightness can be conceptualized as a condition of expanded weakness coming about because of a decrease in physiological save and capacity across various organ frameworks, to such an extent that the capacity to withstand stressors is disabled. Different investigations have shown a solid relationship between slightness and unfriendly peri-employable results. Fragility might be evaluated utilizing different devices; be that as it may, the ideal device for use in a clinical setting still can't seem to be recognized. Notwithstanding the relationship between fragility and unfriendly results, not many mediations have been displayed to further develop results in these patients. ${ }^{13}$ Estimating frailty in more elderly patients with a malignancy might distinguish patients with a greater risk of surgical complications. As it stays disputable how to distinguish slightness, the point of this examination was to think about a pre-employable multi-area delicacy measure dependent on a comprehensive geriatric assessment (CGA) to a changed rendition of the actual aggregate of feebleness (PF) in a companion of more seasoned grown-ups with 
colorectal malignant growth and to investigate the capacity of the two characterizations to predict post-usable complications and endurance. ${ }^{14}$

In the United States, older people account for $15 \%$ of the overall population; this figure is anticipated to rise to 21.4 percent by 2050 . Despite accounting for only $12 \%$ of the population, elderly people accounted for $35 \%$ of all hospital admissions in 2012. In addition, this cohort had a substantially longer average hospital stay and a higher average cost per visit. ${ }^{15}$ Despite the lack of a gold standard operational definition, frailty may be thought of as a condition of heightened vulnerability caused by a decrease in physiological reserve and function across many organ systems, resulting in a reduced ability to tolerate stress. A number of studies have found a substantial link between frailty and poor perioperative outcomes. ${ }^{15}$ Multiple methods may be used to measure frailty; however, the best instrument for use in a clinical context has yet to be found. Despite the link between frailty and poor outcomes, Oakland et al. found that few treatments have been demonstrated to enhance outcomes in these individuals. ${ }^{15}$ Understanding the relationship between surgical morbidity and frailty is precluded by the diversity in the assessment of frailty. ${ }^{7}$ The diversity of surgical intervention in frail patients is of considerable interest. ${ }^{10}$ The majority of studies done on frailty have focused on malignancies, therefore making generalized conclusions is unfair. ${ }^{9,12}$ The usefulness of EFS was implanted as an instrument by which can add prognosis of the surgical outcome on frail patients. ${ }^{7,8}$ The Operative Severity Score (OSS) developed by Shinall and colleagues, also aids as an instrument for a surgical prediction on the frail patients in terms of mortality and independence loss after surgical intervention. ${ }^{2,7,9}$ Patients who have higher dependency before surgical procedure have three times greater chances of death after surgery as compared to those who have no LOI before surgery, therefore, this study was aimed at determining the preoperative frailty assessment screening on the postoperative morbidity and loss of independence (LOI) after surgical procedures.

\section{MATERIALS \& METHODS}

\section{Study Design \& Settings}

A cross-sectional study was conducted from May 2018 to January 2020 and included patients 65 years or older $(n=100)$ who were subjected to frailty assessment before surgery. The patients were enrolled from the Islamabad International Hospital and Research Center (IIHRC).

\section{Data Collection}

The data was collected on the following variables: age, sex, and BMI (body mass index). The data on risk stratification was collected through evaluation of the Edmonton Frail Scale (EFS) and Operative Severity Score (OSS). The surgical procedure was categorized on operative severity score, and Hierarchical Condition Category (HCC).

Table 1: Edmonton Frail Scale.

\begin{tabular}{|c|c|c|}
\hline $\begin{array}{l}\text { The Function of LOI } \\
\text { (Loss of Independence) }\end{array}$ & $\begin{array}{l}\text { Clinical Expression/ } \\
\text { Domain of LOI }\end{array}$ & Scores \\
\hline $\begin{array}{l}1 \text { - Impairment of } \\
\text { Cognition }\end{array}$ & $\begin{array}{l}\text { Unable to draw a } \\
\text { clock, forgetting } \\
\text { important tasks to do, } \\
\text { like forgetting to take } \\
\text { medicine on time. }\end{array}$ & 2 Points \\
\hline 2 - Dependent & $\begin{array}{l}\text { Unable to perform } \\
\text { daily routine activities. }\end{array}$ & 2 Points \\
\hline 3 - Depression & Lack of feelings & 1 Point \\
\hline 4- Mobility & Get-up and go activity & 1 Point \\
\hline $\begin{array}{l}\text { Restrictions } \\
5 \text { - Loss of Weight }\end{array}$ & $\begin{array}{l}>10 \text { seconds } \\
\text { A recent decrease in } \\
\text { lean body weight and } \\
\text { loose clothes }\end{array}$ & 1 Point \\
\hline
\end{tabular}

\section{Edmonton Frail Scale (EFS)}

The presence of geriatric syndromes, chronic 
illness, nutrition, cognition, and independent capacity was evaluated for the study group of old frail patients. Frailty was considered when EFS was 6 or more. The following parameters were incorporated as shown in Table 1.

\section{Operative Severity Score (OSS)}

The Operative Severity Score (OSS) is categorized from 1 to 5 , which is based on the experience of patient physiologic response to surgical trauma (Table 2).

Table 2: Operative Severity Scores (OSS).

\begin{tabular}{|c|c|}
\hline Procedure & Minor, moderate, major, major+ \\
\hline $\begin{array}{l}\text { Number of } \\
\text { Procedures }\end{array}$ & $1,2,2+$ \\
\hline Loss of Blood (ml) & $100,101-500,501-999,>1000$ \\
\hline Malignancy & Present / Absent \\
\hline ype of surgery & $\begin{array}{l}\text { Immediate emergency } 2 \text { hours within } \\
\text { the presentation, emergency more } \\
\text { than } 2 \text { hours after the presentation, } \\
\text { Elective surgical intervention. }\end{array}$ \\
\hline
\end{tabular}

\section{Measurement of the Outcome}

The loss of independence (LOI) and mortalities was evaluated for frail patients, meeting the inclusion criteria. Patients who were referred to skilled rehabilitation centers instead of home, after surgical intervention meet the criteria of LOI. Patients who died postoperatively within 30 days from the same surgery were placed in the definition of mortality, from the same surgical procedure.

\section{Statistical Analysis}

All the data was evaluated in SPSS version 25 for the calculations of frequencies, descriptive statistics, and statistical significant/insignificant differences. The functional dependency was used for patient stratification and analysis. The Chisquare test was applied to see the significant/ insignificant differences between different stratified groups of patients. A p-value of less than 0.050 was considered significant. Odds ratios were also calculated via. Chi-square test.

\section{RESULTS}

A total of 100 patients age 65 years and older were included in this study.

\section{Age Distribution}

The mean age of the patients was 68 years. The maximum age was 95 years, whereas the minimum age was 65 years.

\section{Gender Distribution}

There were $63 \%$ male and $37 \%$ female patients.

\section{Dependency Status Concerning Demographics and Clinical Assessment Loss of Independence - Age Wise}

Maximum patients (78\%) who had no LOI were in age less than 80 years. $11 \%$ of patients who had LOI were only $11 \%$. See Table 3 for details. There existed a significant difference ( $p$-value: 0.004) between age groups (greater than/less than 80 years) of patients and presence/absence of loss of independence (LOI).

Table 3: Loss of independence, age - wise percentage $(n=100)$.

\begin{tabular}{|c|c|c|c|c|}
\hline Age & $\begin{array}{c}\text { No LOI } \\
\text { (n\%) }\end{array}$ & $\begin{array}{l}\text { LOI } \\
\text { (n\%) }\end{array}$ & $\begin{array}{l}\text { Chi- } \\
\text { Square }\end{array}$ & P-value \\
\hline$<80$ & 78 & 6 & & 0.00473 \\
\hline$>80$ & 11 & 5 & 1.9183 & (significant) \\
\hline
\end{tabular}

\section{Body Mass Index (BMI)}

Table 4 shows the stratifications for BMI $\left(\mathrm{kg} / \mathrm{m}^{2}\right)$ groups (less than 25 \& greater than 25) in patients with or without loss of independence (LOI). Maximum patients (88\%) were having $\mathrm{BMI}$ 
$>25$ and among them, maximum patients (81\%) were found without LOI. There existed an insignificant difference ( $p$-value: 1 ) between BMI groups and the existence/nonexistence of Loss of independence (LOI).

Table 4: Information on BMI $(n=100)$.

\begin{tabular}{cccccc|}
$\begin{array}{c}\text { BMI } \\
\left(\mathbf{k g} / \mathbf{M}^{2}\right)\end{array}$ & $\mathbf{n} \%$ & $\begin{array}{c}\text { With } \\
\text { LOI } \\
\mathbf{( n \% )}\end{array}$ & $\begin{array}{c}\text { Without } \\
\text { LOI } \\
\mathbf{( n \% )}\end{array}$ & $\begin{array}{c}\text { Chi- } \\
\text { Square }\end{array}$ & p-value \\
$<25.0$ & 12 & 3 & 9 & & 1 \\
$>25.0$ & 88 & 7 & 81 & 0 & (insignificant) \\
\hline
\end{tabular}

\section{Edmonton Frail Scale (EFS)}

The EFS was between $2-5$ with a mean of 3 . After EFS assessment, $32 \%$ of patients had limited mobility, 22\% had weight loss, $19 \%$ reported depression, $9 \%$ of patients were socially dependent and $18 \%$ reported cognitive impairment (Table 5).

Table 5: Edmonton Frail Scale $(E F S)(n=100)$.

\begin{tabular}{lc|} 
Domain & $\mathbf{n} \%$ \\
Cognitive impairment & 18 \\
Social dependence & 9 \\
Depression & 19 \\
Weight loss & 22 \\
Limited mobility & 32 \\
\hline
\end{tabular}

\section{Operative Severity Score (OSS)}

$65 \%$ of the procedures were elective in patients with an average duration of 150 minutes (100 300 minutes). In $40 \%$ of patients, the OSS was 3 or higher, and $60 \%$ had OSS between $1-2$. Post surgically, $15 \%$ of patients experienced a loss of dependence (Table 6). The OSS was higher in patients with LOI as compared to patients without LOI. Moreover, those patients with a longer stay in the hospital had more LOI compared to a patient with less hospital stay (6 days vs. 1 day). The patients with LOI had higher mortality than those without LOI (11\% vs. $4 \%$ ).
Table 6: Operative Severity Score (OSS) $(n=100)$.

$\begin{array}{cc}\text { Score } & \mathbf{n} \%) \\ 1 & 25 \\ 2 & 35 \\ 3 & 34 \\ 4 & 4 \\ 5 & 2\end{array}$

\section{Postsurgical Risk Factors Based on EFS and OSS}

The patients with EFS $>5$ and OSS $>3$ had more LOI. Also, EFS analysis resulted that those patients with restricted mobility, depression, and dependent socially had increased LOI. These results suggest that frail patients older than 78 years and OSS > 3 had a 39 - fold increased risk of postsurgical LOI. 9 - Fold increased mortality was reported with high EFS with loss of weight and restricted mobility.

\section{DISCUSSION}

The process of aging and disability is a longrecognized process in human history, which is experienced by the old age population. ${ }^{1,3}$ The process of disablement can be described physiologically, how the body coup with an acute and chronic condition that specifically affects a particular system. ${ }^{7,9}$ Surgery performed on old frail patients further aggravated disablement, and surgery is one of the main environmental stresses that increased morbidity among old age population. ${ }^{4,5}$ Surgical intervention poses a challenge for frail patients, to tackle stress, as they are already compromised., 1,3,7 The poor surgical outcome is inevitable in frail patients if not addressed in a multidisciplinary way, and geriatric medicine should actively be involved in the surgical decision-making. Prognosticating LOI can be done at discharge and to deal with chronicity of the problem need specialized rehabilitation centers, unfortunately scarcely available in our country. ${ }^{5,6}$ The purpose of the 
current study was to determine the impact of preoperative frailty assessment screening on postoperative morbidity and loss of independence (LOI). Singed's frailty aggregate or REFS (Reported Edmonton Frail Scale) can be utilized as a screening instrument to recognize slightness in more seasoned inpatients in Vietnam and foresee mortality. Frailty screening can help with zeroing in on assigned meagerness redid drugs, similar to food, early flexibility, and remedy review, for these weak patients to chip away at clinical outcomes as researched by Nguyen et al. ${ }^{16}$ The majority of our patients (88\%) had a BMI more than 25 , and the majority of them (81\%) did not have an LOI. The EFS ranged from 2 to 5 , with a mean of 3 . Following EFS evaluation, 32\% of patients indicated restricted mobility, 22\% reported weight loss, 19\% claimed despair, 9\% were socially dependent, and $18 \%$ had cognitive impairment. 40 percent of patients had an OSS of 3 or higher and 60 percent had an OSS of $1-2$. After surgery, $15 \%$ of patients reported a loss of independence. Those with LOI had a greater OSS than patients without LOI. Furthermore, patients who were in the hospital for a longer period had greater LOI than patients who stayed for a shorter period. We found that the patients with EFS greater than 5 and OSS greater than 3 had a higher LOI. Additionally, EFS analysis revealed that patients with limited mobility, melancholy, and social dependency had higher LOI. According to these findings, fragile individuals over the age of 78 with an OSS greater than 3 had a 39-fold higher incidence of postsurgical LOI. High EFS with weight loss and limited mobility was associated with a 9-fold increase in death.

Patients with LOI at discharge, more than half will experience LOI after the 30 days. $^{7}$ It was demonstrated by Roo et al, that surgical intervention on old patients, 33\% decline in already compromised physiology is inevitable after 1 year. ${ }^{7,9}$ LOI after the surgical intervention was addressed by utilizing EFS, SSC. EFS 6 and higher were given special attention to be assessed by the geriatric practitioner for risk stratification and LOI post operatively. ${ }^{12}$ As reported by other studies that EFSs more than 6 were found significantly at risk for LOI. ${ }^{11,12}$ Multiple risks are not the same, however, frailty increases morbidity and mortality on many risky surgeries like aneurysms, tumors, etc. ${ }^{7,9}$ Frail patients need to be screened by the available system of history, physical examination morbid findings, frailty assessment tools like EFS, FSS. ${ }^{1,5,6}$ Their overall screening will help in the allocation of resources and preoperative coordinated care. ${ }^{7,8}$ Donald et al, successfully incorporated the coordinated Clinical Frailty Scale into preoperative frail old patients. Our results were significantly predicting post-surgical morbidity and mortality. 9,12 Kumar et al. also reported high readmission and morbidity post-surgically on frail old age patients. By utilizing a scoring system of $\mathrm{EFS}>6, \mathrm{HCC}>1$, and OSS $>3$, there was a 15 percent increase in the overall LOI. ${ }^{1,6,8}$ Most frail patients age more than 80years with EFS $>6$, who underwent a stressful physiologic procedure with OSS > 3, there was 40 fold increase in the LOI postoperatively. ${ }^{5,11}$ These results suggested that knowing the status of frailty, and incorporating global operative scores gives surgeons important predictive information which helps with surgical decisions and TGO allocate proper rehabilitation center postoperatively., Hirvensalo et al, conducted a study on old dwelling communities and found a $3-5$ fold increase in LOI in their study population. ${ }^{5}$ Lacroix et al, conducted a similar study, and they found good functional outcomes in physically active individuals despite their chronicity of the disease. Less mobile patients with a sedentary lifestyle have more LOI than physically active ones. ${ }^{7}$ Their work suggested an optimization program for the optimization of mobility before surgeries of old frail patients. ${ }^{3,11,12}$

Hui-Shan Lin et al, $^{17}$ studied patients ranging in age from 75 to 87 yearsold who had cardiac, oncological, general, vascular, and hip fracture procedures. Twenty-one different devices were 
used to assess frailty. Frailty was observed to predict postoperative mortality, comorbidities, and a longer length of stay in elderly and oldestold surgical patients. Various frailty instruments may be better suited to the acuity and kind of surgical patients. There is a need for more research on the relationship between frailty and post-surgery return to pre-morbid function, discharge destination, and quality of life. Meyers et al, $^{18}$ included 118 patients, among them there were $50 \%$ patients who had an EFS score of $0-3$, $28.8 \%$ had an EFS score of $4-6$, and $20.8 \%$ had an EFS score $\geq 7$. Frailty is a strong and independent prognostic factor for all-cause mortality in old patients giving ACS (acute coronary syndrome) as concluded by Blanco et al. $^{19}$ There was no connection between the EFS and receipt of chemotherapy for the examination population; nonetheless, avoidance of stage II patients showed a diminished probability of getting chemotherapy with higher EFS scores according to Meyers et al. ${ }^{18}$

The exploratory examination recommends that EFS can recognize patients that oncologists might have thought were excessively fragile for chemotherapy, autonomous of PS. Consequently, the EFS can add a reproducible, and quantifiable proportion of feebleness to the clinician's dynamic toolset. A subsequent report will use the EFS continuously and decide whether it can limit spontaneous medical services for chemotherapy patients as worked by Meyers et al. ${ }^{18}$ The examination proposes that the EFS could give a reproducible, quantifiable proportion of fragility which, when combined with clinical judgment, may upgrade the dynamic cycle much further. Future work might analyze whether usage of the EFS can help clinicians and patients in examining therapy alternatives, recovery, and spontaneous medical care use in older disease patients contrasted with clinical judgment alone. Meyers et $\mathrm{al}_{1}{ }^{18}$ found it appropriate to decide if intercessions to address frailty in older patients can further develop resilience to chemotherapy. There was no verifiable connection found by Keenan et $\mathrm{al}_{,}{ }^{20}$ between EFS score and the presence of grade 3 or 4 toxic substance levels. This assessment showed that neither EFS score, age nor ECOG performance status were farsighted of radiotherapy hurtfulness, breaks in therapy, or clinical facility certifications. Patients with esophageal cancer, gynecological cancers, and cell breakdown in the lungs had a high speed of destructiveness and center affirmation, which may include the prerequisite for additional arrangement help in these social affairs. While comprehensive geriatric assessment is recommended, further assessment of old patients for risk of toxin levels from radiotherapy is mandatory as suggested by Keenan et al. ${ }^{20}$

This present study is very educational for medical assistants to learn the art of implementing EFS scoring, because, unfortunately in our country, many centers cannot assess medical assistance performance to implement the EFS scoring system and OSS.

\section{CONCLUSION}

Higher patient dependency before surgery corresponds to poor outcomes compared to patients who do not have a loss of their independence before surgery. This is a novel study for our country to implement EFS, HCC, and OSS to surgical old frail patients preoperatively. This helps to identify risky old frail patients.

\section{RECOMMENDATIONS}

Pre-operative assessment of patients with OSS and EFS may help in predicting patients' morbidity and mortality after surgery. Further studies on these risky old patients are needed to implement the standard recommendation to preempt morbidity and mortality. 


\section{LIMITATION}

This was a uni-central and generalized study with regards to the general outcome of patients undergoing a wide variety of surgeries, not focused on a single type of surgery.

\section{REFERENCES}

1. Yii MK, Ng KJ. Risk-adjusted surgical audit with POSSUM scoring system in a developing country. Physiological and operative severity score for the enumeration of mortality and morbidity. Br Surg. 2002; 89: 110-3.

2. Treharne GD, Thompson MM, Whiteley MS, Bell PR. Physiological comparison of open and endovascular aneurysm repair. Br J Surg. 1999; 86: 760-4.

3. Berian J, Zhou L, Russell $M$, et al. Postoperative delirium as a target for surgical quality improvement. Ann Surg. 2018; 268: 93-99.

4. Donald G, Chaffarian A, Isaac F. Preoperative frailty assessment predicts loss of independence after vascular surgery. J Vasc Surg. 2018; 68: 1382-1389.

5. Mehanty S, Rosenthal R, Russel M. Optimal perioperative management of the geriatric patients: a best practices guidelines from the American College of Surgeons NSQIP and the American Geriatric Society. J Am Coll Surg 2016: 157-173.

6. Engelhardt K, Reuter Q, Liu J. Frailty screening and a frailty pathway decrease length of stay, loss of independence, and 30-day readmission rates in frail geriatric trauma and emergency general surgery patients. J Trauma Acute Care Surg. 2018; 85: $167-173$.

7. Stoica N, Baddigam R, Wajahn J. The gap between clinical research and standard of care: a review of frailty assessment scales in perioperative surgical settings. Front Public Health, 2016; 67: 157-173.

8. Verbrugge LM, Jette AM. The disablement process. Soc Sci Med. 1994; 38: 1-14.

9. US Census Bureau. National Population Projections Report. Washington, DC: US Census Bureau, 2017.

10. Dall RM, Gallo PD, Chakradarti R. An aging population and growing disease burden will require a large and specialized health care workforce by 2025. Health Aff (Millwood), 2013;
32: 2013-2020.

11. Etzioni DA, Liu JH, O'Connell JB. Elderly patients in workloads: a population based analysis. Am Surg 2004: 691-95.

12. LaCroix AZ, Guralnik JM, Berkman LF, et al. Maintaining mobility in late life II. Smoking, alcohol consumption, physical activity and body mass index. Am J Epidemiol. 1993; 137: 858-869.

13. Richards SJ, Frizelle FA, Geddes JA, Eglinton TW, Hampton MB. Frailty in surgical patients. International Journal of Colorectal Disease, 2018; 33 (12): 1657-66.

14. Kristjansson $S R$, Rønning $B$, Hurria $A$, Skovlund $E$, Jordhøy MS, Nesbakken A, Wyller TB. A comparison of two pre-operative frailty measures in older surgical cancer patients. Journal of Geriatric Oncology, 2012; 3 (1): 1-7.

15. Oakland K, Nadler R, Cresswell L, Jackson D, Coughlin PA. Systematic review and meta-analysis of the association between frailty and outcome in surgical patients. The Annals of the Royal College of Surgeons of England, 2016; 98 (2): 80-5.

16. Nguyen AT, Nguyen TX, Nguyen TN, Nguyen $T H$, Pham T, Cumming R, Hilmer SN, Vu HT. The impact of frailty on prolonged hospitalization and mortality in elderly inpatients in Vietnam: A comparison between the frailty phenotype and the Reported Edmonton Frail Scale. Clinical Interventions in Aging, 2019; 14: 381.

17. Lin HS, Watts JN, Peel NM, Hubbard RE. Frailty and post-operative outcomes in older surgical patients: a systematic review. BMC Geriatrics, 2016; 16 (1): $1-2$.

18. Meyers BM, Al-Shamsi HO, Rask S, Yelamanchili R, Phillips CM, Papaioannou A, Pond GR, Jeyabalan N, Zbuk KM, Dhesy-Thind SK. Utility of the Edmonton Frail Scale in identifying frail elderly patients during treatment of colorectal cancer. Journal of Gastrointestinal Oncology, 2017; 8 (1): 32.

19. Blanco $S$, Ferrières J, Bongard V, Toulza $O$, Sebai $F$, Billet $\mathrm{S}$, Biendel $\mathrm{C}$, Lairez $\mathrm{O}$, Lhermusier $\mathrm{T}$, Boudou $\mathrm{N}$, Campelo-Parada F. Prognosis impact of frailty assessed by the Edmonton Frail Scale in the setting of acute coronary syndrome in the elderly. Canadian Journal of Cardiology, 2017; 33 (7): 9339.

20. Keenan LG, O'Brien M, Ryan T, Dunne M, McArdle O. Assessment of older patients with cancer: 
Edmonton Frail Scale (EFS) as a predictor of adverse outcomes in older patients undergoing radiotherapy. Journal of Geriatric Oncology, 2017; 8 (3): 206-10.

\section{Additional Information}

Disclosures: Authors report no conflict of interest.

Ethical Review Board Approval: The study was conformed to the ethical review board requirements.

Human Subjects: Consent was obtained by all patients/participants in this study.

\section{Conflicts of Interest:}

In compliance with the ICMJE uniform disclosure form, all authors declare the following:

Financial Relationships: All authors have declared that they have no financial relationships at present or within the previous three years with any organizations that might have an interest in the submitted work.

Other Relationships: All authors have declared that there are no other relationships or activities that could appear to have influenced the submitted work.

\section{AUTHORS CONTRIBUTIONS}

\begin{tabular}{|l|l|l|}
\hline Sr.\# & Author's Full Name & Intellectual Contribution to Paper in Terms of: \\
\hline 1. & $\begin{array}{l}\text { Shams-ud-Din } \\
\text { Ishfaq Ahmed }\end{array}$ & Study design and methodology. \\
\hline 2. & $\begin{array}{l}\text { Shams-ud-Din } \\
\text { Ishfaq Ahmed }\end{array}$ & Paper writing, referencing, and data calculations. \\
\hline 3. & Shams-ud-Din & Data collection and calculations. \\
\hline 4. & Ishfaq Ahmed & Analysis of data and interpretation of results etc. \\
\hline 5. & Ishfaq Ahmed & Literature review and manuscript writing. \\
\hline 6. & $\begin{array}{l}\text { Shams-ud-Din } \\
\text { Ishfaq Ahmed }\end{array}$ & Quality insurer. \\
\hline
\end{tabular}

